\title{
Lethal hemothorax following thoracic dislocated spinal fracture: the usefulness of arterial embolization
}

\author{
Seiji Morita • Tomoatsu Tsuji • Takeshi Yamagiwa • \\ Tomokazu Fukushima $\cdot$ Sadaki Inokuchi
}

Received: 17 April 2009 / Accepted: 19 May 2009/Published online: 1 August 2009

(C) Springer-Verlag London Ltd 2009

\begin{abstract}
A 68-year-old male fell from a height of $3 \mathrm{~m}$. He was conscious and alert, and complained of back pain, dyspnea, and paraplegia with hemodynamic instability. Initial axial and three-dimensional computed tomographic (CT) scans revealed a massive hemothorax on the right side and a dislocation (Th11) of the thoracic bamboo spine (Fig. 1a, b). We believed that the cause of the hemothorax was from the injured intercostals artery following the dislocation of the thoracic spine; therefore, angiography was performed immediately to isolate the source of bleeding. Angiographic findings revealed extravasation of the contrast medium from the intercostal artery (Th11; Fig. 1c). Selective arterial embolization (AE) was performed with gelatin sponge particles. After AE, the extravasated contrast medium disappeared, and his hemodynamic state stabilized. On the 16th day of hospitalization, the thoracic spine fracture was
\end{abstract}

successfully repaired, and he was kept at the hospital to recuperate.

Life-threatening hemothorax is uncommon following a thoracic vertebral fracture $[1,2]$. There are only a few reports on life-threatening hemothorax following a thoracic vertebral fracture; surgical management was adopted in these cases $[1,2]$. However, treatment for life-threatening hemorrhage following fractures of the spine has not yet been established. This is the first successful treatment report on life-threatening hemothorax following thoracic vertebral fracture. We thought that $\mathrm{AE}$ was a good choice of treatment for our patient. However, this method could not be useful if the angiographic finding showed the extravasation of the contrast medium from the great anterior radicular artery (artery of Adamkiewicz) because of spinal infarction. Therefore, the method of management should be changed immediately.

S. Morita $(\bowtie) \cdot$ T. Tsuji $\cdot$ T. Yamagiwa $\cdot$ T. Fukushima $\cdot$

S. Inokuchi

Department of Critical Care and Emergency Medicine,

Tokai University School of Medicine,

143 Shimokasuya Isehara-City,

Kanagawa, Japan 2591193

e-mail: morita@is.icc.u-tokai.ac.jp 
Fig. 1 (a) Axial thoracic CT scan. This CT scan shows massive hemothorax on the right side. (b) Three-dimensional vertebral CT scan. This CT shows a dislocation (Th11) of the thoracic bamboo spine. (c) Right internal arteriogram (Th11). Arteriogram shows extravasation of the contrast medium in the intercostal artery

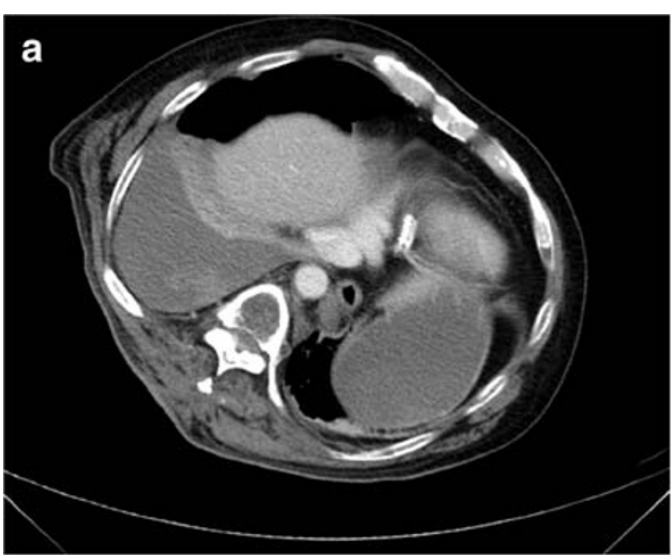

\section{b}

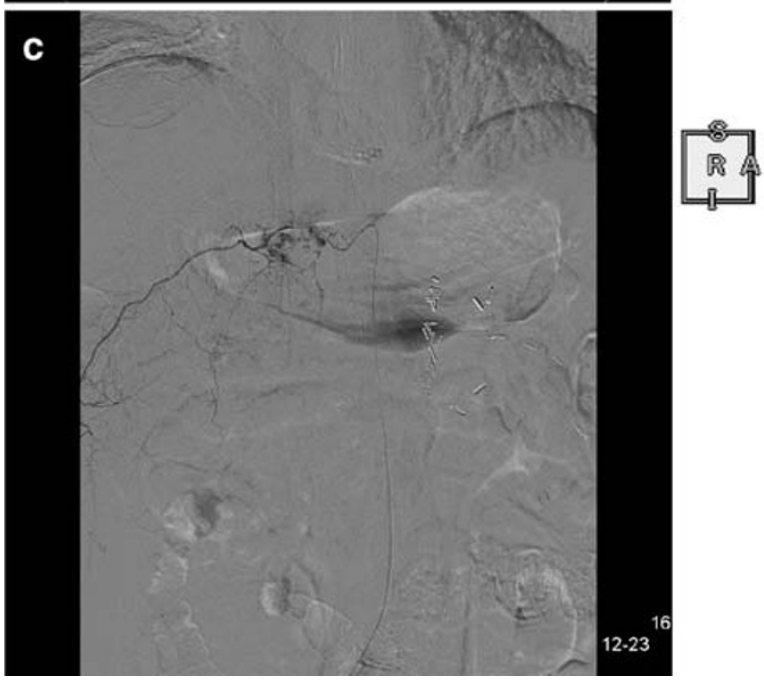

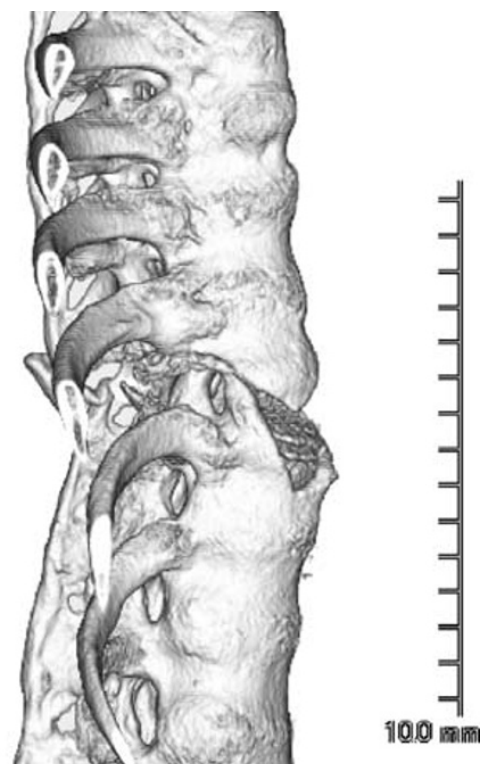

Conflicts of interest We have no financial or proprietary interest in the subject matter or materials discussed in the manuscript, including employment, consultancies, stock ownership, honoraria, or paid expert testimony.

\section{References}

1. van Raaij TM, Slis HW, Hoogland PH et al (2000) Massive haemothorax following thoracic vertebral fracture. Injury 31:202-203

2. Dalvie SS, Burwell M, Noordeen MH (2000) Haemothorax and thoracic spinal fracture: A case for early stabilization. Injury $31: 269-270$ 